\title{
Spontaneous Self-Repairs in Speech: Processes and Representations
}

Willem J.M. Levelt

Nijmegen, the Netherlands

\section{Phonetics, Psycholinguistics, and Self-Repairs}

Psycholinguistics study the acquisition, comprehension and production of language, but the study of language production has not advanced at the same pace as the other two main areas of inquiry. While there are notable exceptions, the impetus to study the process of speaking does not usually come from within the discipline itself, but from neighboring fields, such as ethnomethodology, aphasiology, and last but not least, phonetics. No approach has informed the psychological study of language production to the same extent as the systematic analysis of spontaneous speech errors, a technique which was reintroduced by Cohen and Nooteboom during the sixties. Traffic in the reverse direction has been light: the psychology of speaking has not influenced phonetics to the same degree.

It is only honest, therefore, to speak about the potential contributions to phonetics of psycholinguistic production research. The study of spontaneous self-repairs may well develop into such a contribution. Self-repairs are, on first view, rather complex phenomena. And surely, they involve quite disparate phonetic processes, such as self-monitoring, the production and detection of phonetic, lexical and other types of speech errors, self-interruption, prosodic marking of the correction, etc. This complexity on the surface, however, does not preclude systematicity at a deeper level, a systematicity which may reveal principles of organization of the speech production process that would be hard to discover on the basis of laboratory data alone.

The more specific psycholinguistic contribution here is to clarify the character of this underlying systematicity. The psycholinguist will, more in particular, try to analyse the levels of representation involved in the generation of a speech repair. What are the relevant entities for the analysis of a speaker's self-monitoring, self-interruption, re-starting, etc? Are they phonetic features, phonemes, words, clauses, concepts, intentions, or several of these at the same time? And closely related to this is the issue of how these entities are stored and addressed during the process of repairing. What sort of memory structures are involved, for instance, in repeating part of the same utterance, or in aligning the prosody of the correction to that of the interrupted utterance? The psycholinguist will try to explain self-repairs in terms of the same representations and processes which underly normal fluent speech. 
Phonetic complexity may turn out to be psycholinguistic systematicity. Let us therefore turn to normal fluent speech first.

A speaker usually constructs and uses an utterance in order to realize an intention, such as to inform or convince an interlocutor, to request action or information from him, etc. The psycholinguist tries to follow the flow of information from the initial conception of an intention to the articulation of speech. The processing is done in stages involving different levels of representation. An early stage concerns the retrieval and selection of information whose expression may fulfill the intention. The speaker takes a certain perspective with respect to this information; the information will have a focus, it may contrast with earlier expressed information or rather be a further elaboration of what was previously said, there will be a spatio-temporal relation between the speaker and the information selected, and the speaker will have an attitude of belief, want, distrust etc. with respect to that information. The information selected for expression is usually called the message. The subsequent levels of representation result from mapping the message onto linguistic form. Major processes here are the retrieval of lexical items, the creation of syntactic configurations such as phrases and clauses, the realization of morphonological structure. The final output of these processes can informally be called inner speech. Phenomenologically speaking, inner speech is a level of representation; whether it can be used as a theoretical construct is still to be seen.

The last stage in the flow of information is the planning and execution of articulatory procedure. Its output is overt speech.

How do the observable phenomena in self-repair proceed from different levels or types of representation involved in normal speech? In the following, I will discuss some of these issues in the light of a corpus of 959 tape-recorded repairs. The corpus was obtained in an experiment designed for a different purpose. In this experiment 53 subjects were asked to describe spatial patterns consisting of colored dots, connected by horizontal or vertical arcs, such as the ones in Figure 1. There was, on the average, one repair in every third pattern description.

The main analysis of this corpus of repairs can be found in Levelt (1983), a subsequent study of prosodic aspects of these repairs appears in Levelt and
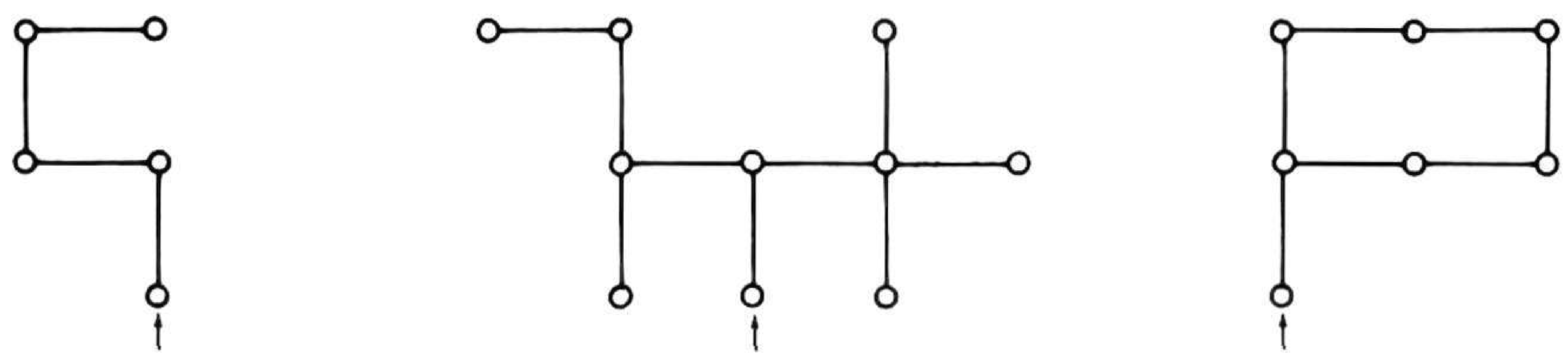

Figure 1. Examples of patterns described by subjects. Dots in the patterns were differently colored. 
Cutler (1983). Others, especially Ewald Lang (unpublished) have also contributed to the analyses of this corpus.

\section{The Structure of Repair}

A repair consists, typically, of three phases. In the first, trouble occurs and is detected by the speaker, who decides to interrupt the flow of speech. The segment of speech affected will be called the original utterance. In example (1) the original utterance is 'right of pink is a black'.

1. right of pink is a black, er a blue point

The original utterance contains a lexical error ('black' for 'blue') which is apparently detected by the speaker, who interrupts immediately after the error.

The second phase is one of filled or unfilled pausing. In (1) the pausing is filled by 'er', but other editing terms are also frequently used. They are systematically related to the source of trouble, and how recently it occurred. The third and last phase consists of the correction itself. In (1) it is 'a blue point'. Important events take place in this phase. The speaker tells the listener how to relate the repair to the original utterance, by restricted syntactic, lexical, and prosodic means. In this way the speaker establishes on-line interpretability of the repair for the listener.

Let us now turn to these three phases in some more detail.

\section{I.Interrupting the Utterance}

There are many possible reasons why a speaker might want to interrupt the flow of speech, but two major sources of trouble were found in the corpus of self-repairs on which the present study is based. The first is the appearance of error, be it a phonetic error (seldom, less than $1 \%$ of the data), a lexical error as in (1) (frequent, $38 \%$ of the corpus), or other (2\%).

The second most frequent situation is one in which, although what was said was correct, it was not fully appropriate. An example is given in (2):

2. a line to the yellow disc, to a yellow disc

Here the yellow disc had not been introduced before by the speaker, and it is thus more appropriate to use the indefinite article.

Another appropriateness repair is given in (3):

3. right thereof, of the orange one a blue dot

The demonstrative "thereof refers to a dot mentioned in a previous utterance, but the speaker realizes that there may be an ambiguity of reference here, and decides to name the referent explicitly. In neither (2) nor (3) 
anything was said that was false. The new utterance is only more appropriate in the discourse context (the precise criteria for this category of repairs are outlined in Levelt, 1983). Thirty percent of the corpus consists of appropriateness repairs. These two main categories of trouble, error and inappropriateness, are very different in their consequences for the way in which the repair is made. It should be added, for completeness' sake, that there is a third major category of repairs in the corpus whose etiology cannot be decided unambiguously; we called them covert repairs or hesitations. An example is given in (4):

\section{4. up is, er blue}

It is unclear whether the speaker had a perceptual problem, had difficulty finding the color name, intercepted an erroneous lexical item that he was about to pronounce, or otherwise. These covert repairs account for $25 \%$ of the corpus. (the remaining $4 \%$ of the corpus consists of minor categories which are of no interest for the present purposes).

How does the speaker detect trouble? Or in the above terminology: which levels of representation are accessible to the speaker? My conjecture is that the speaker can attend to messages, to inner speech and to overt speech, but to nothing else. He has no direct way of monitoring his own lexical access procedures, the construction of phrasal configurations, the assignment of syntactic agreement, the construction of tone groups, the generation of coarticulation, etc. The speech production apparatus is cognitively impenetrable, to use Pylyshyn's (1980) terms. If this is correct, there are exactly three levels of representation which allow for monitoring. The first one is the message level. A speaker may want to replace or change a message before it enters the next stage of processing. This may lead to delays or hesitation, such as in (4).

The second one is at the level of 'inner speech', and the third one is at the level of overt speech. For the latter two levels I suppose that the speaker uses his normal speech perception apparatus; he or she will parse the speech produced, and derive the message, as if listening to someone else. Self-monitoring will in the first place consist of comparing this derived message to the intended message. If there is a major or communicatively important difference in truth, reference, clarity, etc. between derived and intended message, the speaker may want to interrupt speech and make a correction. The speaker can also monitor for certain well-formedness aspects of the self-produced speech, such as phonetic errors. Although the processes involved are quite mysterious, they need not be different from those involved in detecting ill-formedness in the speech of others.

The moment of interruption can vary widely with respect to the trouble spot. In (1) it follows the trouble item immediately, and there are many instances in the corpus where interruption is even faster, namely within the trouble item itself, as in example (5) below. One major theoretical issue is 
why speakers do not always interrupt immediately. In (2), the trouble item 'the' is followed by two more words before interruption. Is this due to inertia in the production apparatus, i.e. a tendency to complete some linguistic unit (a clause, a phrase, a tone group)? Or is it rather the case that the speaker did not detect the trouble until two words later?

The analyses point largely to the latter explanation. We obtained statistical evidence for a slight tendency on the part of the speakers to interrupt more often (in $71 \%$ of the cases) at the end of a surface phrase, such as an NP, a VP, a PrepP, than was to be expected statistically (we found that a random point of interruption in these pattern descriptions completed a phrase in $58 \%$ of the cases). The inertia theory predicts that a speaker tends to complete a phrase after detection of trouble. Delayed interruptions will therefore respect phrase boundaries more often than immediate interruptions. This is, however, not what was found. Phrase structure was respected in $66 \%$ of the delayed interruptions, but in as much as $74 \%$ of the immediate interruptions. This argues against the inertia theory. The tendency to respect constituent structure should rather be interpreted as resulting from a detection mechanism. There is an increased chance of detecting trouble towards the end of a surface phrase, and indeed we found that the rate of detected versus non-detected errors increases sharply towards the ends of phrases (cf. Levelt, 1983). The speaker's attention apparently fluctuates between constructing the message and monitoring the inner or overt speech. Ends of phrases are natural points for checking their contents, or in other words, the phrasal structure of inner and/or overt speech dictates the rhythm of attention shifts. This phrasal constraint is not due to formulating inertia, but to trouble detection, i.e. to perceptual parsing. As far as this goes, we can maintain that speakers interrupt their speech immediately upon detection of trouble. This rule is in full correspondence with Nooteboom's (1980) analysis of the Meringer data. The rule predicts that speech can be stopped at any point after detection of trouble. In one analysis we checked whether a speaker respects phonotactic boundaries while interrupting his speech. There were 172 within-word interruptions in the corpus. An example is given in (5):

\section{5. rechtsaf naar/z/-, wit (right to /b/-, white)}

Here the speaker started saying 'zwart' (black), but then interrupted the incorrect word to replace it by 'wit' (white). The interruption in (5) violates phonological well-formedness in Dutch, /z/ is, phonotactically speaking, not a possible word. We used the 'possible word' criterion to listen to all cases of word-interruption in the corpus. Although such judgments were not equally straightforward, there were 67 cases where we felt certain that the interrupted fragment was phonotactically not a possible word of Dutch, as in (5). It is hard to evaluate this finding statistically, but one thing can be said with confidence: nothing prevents the speaker from interrupting speech at phonologically odd places. Again, inertia of the production apparatus, in 
this case at a phonotactic level of representation, does not seem to be an important determinant of moments of interruption.

What we did find, however, is that speakers tend to complete words before interruption. Only $20 \%$ of appropriateness and error repairs involve halting within a word. But there is an interesting rule here; these $20 \%$ are not evenly distributed over repairs. Of the appropriateness repairs only $10 \%$ involve word interruption, whereas $28 \%$ of error repairs do. Is it the case that speakers are willing to interrupt an erroneous word, but not a correct (though potentially inappropriate) one? This is easily checked. It predicts that the percentage of word interruption should be high for immediate halts in error repair, i.e. halts during or right after the trouble item such as in (1) and (5) above; in these cases a within-word interruption is indeed interruption of an erroneous word. The percentage should, however be low for error repairs with delayed interruption, such as (6):

6. and left of the black disc, no right of the black disc...

Here left is erroneous, but the subsequent words till interruption are all correct. If the rule 'Do not interrupt correct words' holds, there should be a low incidence of within-word interruptions in these delayed cases. What we found was $47 \%$ word-interruptions when halting was immediate, against $17 \%$ when halting was delayed in error repairs. This is in agreement with the rule. It should be noted that this qualifies the interruption rule we gave a moment ago: there are cases where a speaker does not immediately interrupt upon detection of trouble. Speakers tend to complete words in all cases, but they are willing to interrupt ones that are erroneous. This finding is in agreement with Nooteboom's (1980) analysis of the Meringer corpus, and one may conclude that the interruption process is sensitive to representations of meaningful words.

\subsection{Editing Terms}

Editing terms vary in the degree of contrast they establish. If the term is 'no', 'rather' ('of' in the Dutch corpus), or 'sorry', it involves an explicit rejection of what was said. But if it is 'therefore' ('dus' in the Dutch corpus) it rather confirms the previous expression. The degree of contrast set up depends on the occasion for repair: error releases much more contrast than does inappropriateness. Indeed we found a much higher incidence of terms such as 'no' 'or', 'sorry' in error repairs than in appropriateness repairs. Inversely, Dutch 'dus' occurred exclusively in appropriateness repairs. More generally, error repairs released more than twice as many editing expressions than appropriateness repairs (62\% versus $28 \%$ ).

The degree of semantic contrast in a repair is not only expressed by the editing term, but also by prosodic features. In a recent paper Cutler (1983) proposed to make a distinction between repairs that are prosodically marked versus those that are unmarked. Pitch, amplitude and relative duration of an 
unmarked repair closely mimic those of the trouble segment. A repair is marked when repair and trouble item differ prosodically. Levelt and Cutler (1983) applied this distinction to a subset of 299 lexical repairs in the present corpus, i.e. repairs where a single trouble word was replaced in the repair. We found that error repairs are far more often prosodically marked (in 53\% of the cases) than appropriateness repairs (only 19\%), and we could show that this is indeed due to the degree of semantic contrast established in the error repairs. Ewald Lang (unpublished) drew our attention to a correlation between editing term used and prosodic markedness. If we compare the 'contrast éstablishing' editing terms 'nee' (no), 'of' (rather), 'sorry' (sorry) to the 'neutral' editing term 'eh' (er) and the non-contrasting 'dus' (therefore), we find $55 \%$ prosodically marked cases among the former repairs, but only $32 \%$ among the latter. Editing terms and prosodic marking thus seem to arise from a semantic level of representation, but this does not exclude the existence of other determinants. It was argued in Levelt (1983) that the interjection 'er' entertains a rather mechanical relation to the interruption process. The faster the interruption after trouble, the higher the incidence of 'er'.

\subsection{The Correction and its Relation to the original Utterance}

There are at least three determinants of the way in which the speaker constructs the correction. I will call them intentional, interactional and structural.

\subsubsection{Intentional Determinants}

The intentional determinants have to do with what a correction is made for, especially whether the speaker intends to patch up an error or rather to find a more appropriate way of expressing the same state of affairs. We found major differences between corrections for error and corrections for appropriateness. I already mentioned the difference in prosodic marking between these two types of repair. A major finding is furthermore that corrections for error are highly conservative, closely copying the wording of the original utterance. This is far less so for appropriateness repairs. A detailed account can be found in Levelt (1983).

\subsubsection{Interactional Determinants}

I will also be short on the interactional determinants. The main point is this: The speaker's sudden interruption of the flow of speech leaves the listener with a so-called 'continuation problem': how is the new utterance to be related to the interrupted utterance? The listener must decide how to 'splice' the two parts together, so to say. We were surprised to find that speakers construct their repairs in such a way that the listener can solve this 'continuation problem' on-line, i.e. no later than upon hearing the very first word of the correction. The main rules the speaker adheres to in order to achieve this for the listener are given on page 48 of the Abstracts of this Congress, and further details are to be found in Levelt (1983) 


\subsubsection{Structural Determinants}

There are restrictions on the ways in which the spcaker can make a repair which are purely structural in character. Example (7) is a well-formed pair:

7. Is he seeing, er interviewing patients?

A grammatically ill-formed way of correcting for the same trouble would be (8):

8. ${ }^{*}$ Is he seeing, er he interviewing patients?

There is nothing semantic or pragmatic which forbids (8), it is fully transparent but still ill-formed. Is this ill-formedness a consequence of the structure of the correction itself, i.e. 'he interviewing patients'? One might argue that it doesn't have the right constituent structure for being used as a correction. But this is not so; the same correction is all right in the following example:

9. Is she, er he interviewing patients?

Apparently, the grammatical well-formedness of a repair is a function of the structural relation between the original utterance and the correction. In Levelt (1983) I have argued that this structural relation is essentially the same as the relation between conjuncts in a coordinate structure, and a precise rule was given how to derive the well-formedness of a repair from the well-formedness of a corresponding coordination. This will not be repeated here. On this occasion I would rather address the issue of how this structural relation is realized in the process of speaking.

The speaker has certain 'grammatical commitments' at the moment of interruption. In (7), for instance, at the moment after 'seeing' there is the commitment to complete the verb phrase by either a noun phrase or a complement clause. Another way of putting this is that the production process is 'under the control of VP' at the moment of interruption. Hoenkamp (1982) suggests that this production process is held 'in suspension' during the editing phase, and subsequently reactivated in order to produce the correction. In other words, there would be a way to store the control structure of the interrupted utterance. This control structure is at a different level of representation than the message. The speaker often changes (adapts, specifies) the message in making a repair, but that change is executed under the same control structure. And if only the message, but not the original utterance itself, were kept in store, one could not prevent a repair such as (8) which is, as was argued, semantically and pragmatically fully transparent.

The suspension theory puts the storage of the relevant features of the original utterance on the output side: the formulation program is in a state of abeyance. But one could also put storage more in the in put side. A possibility suggested by Levelt (1983) is that the speaker, who is his own listener, keeps a 
trace of his own recent inner or overt speech. This trace can then be referred to in the construction of the repair proper. This would involve a very different type of representation. One would either expect the trace to be in echoic memory, which is a rather short term acoustic storage, or in working memory, i.e. it is at least phonemically coded or else semantically.

It is not an easy task to find out which representational system mediates between the structure of the original utterance and the structure of the repair, and I have no definite answer to offer. Still, I feel that a phonetic analysis may clarify these issues. Consider first working memory as the locus of storage. It is well known that the fine acoustic shape of speech is not represented in working memory; the level of coding is phonemic or semantic rather than phonetic. One would therefore not expect the repair to be an acoustic continuation of the interrupted original utterance, though grammatical contiguity as in (7) or (9) would be possible. Echoic memory as the locus of storage leads to a different prediction. In this case there exists a faithful auditory trace, but it is short-lived. The most accurate measurements of 'brief auditory storage' are those by M. Treisman and Rostron (1971); they found that the auditory trace was lost in about $1 \mathrm{~s}$., confirming earlier data for storage of running speech obtained by A. Treisman (1964) who found a value of $1.3 \mathrm{~s}$. One would therefore predict good acoustic contiguity for short delays between trouble item and replacement, but diminishing contiguity for longer delays, with an asymptote at 1 to $1.3 \mathrm{~s}$.

The suspension theory, finally, predicts that acoustically the new utterance should fit seamlessly into the original utterance, even for longer delays (or at least there is no known limit on the persistence of an interrupted speech program).

The obvious phonetic analysis to undertake for distinguishing these three loci of memory is to splice the new utterance, i.e. the correction itself, in to the original interrupted utterance at the appropriate place, that is deleting all the repeated material and the whole editing phase, and then to listen whether the resulting utterance is phonetically natural (I am grateful to Anthony Cohen who suggested this way of splicing to me).

If the locus of storing the original utterance is working memory, naturalness of the spliced utterance will be a matter of accident, since no acoustic or motor information is preserved in working memory. In other words, naturalness will not generally result.

The echoic memory theory predicts naturalness for cases where the repair had a short interval between trouble and replacement. Naturalness will however, break down for cases where that interval exceeded 1 to $1.3 \mathrm{~s}$,

If naturalness is preserved for cases where the interval substantially exceeded the 1 to $1.3 \mathrm{~s}$. limit, the suspension theory is the remaining alternative. It should not be expected, of course, that the interrupted speech program will be preserved indefinitely, and it is an interesting empirical issue to find out what size of interval can be bridged by the program in abeyance. I would, finally, like to express my awareness that the splicing test is not a definitive one in any sense, but it is probably as far as one can get on the basis of natural data. 
We took special care in selecting utterances for this splicing test. It was, firstly, important to take repairs with a fair range of delays between trouble item and replacement. Secondly, the sample should contain both error and appropriateness repairs; they are intentionally quite different, but this should be immaterial for any of the three forms of storage. Thirdly, it was decided to splice both marked and unmarked repairs. Since marking involves a change of the prosodic structure, marked repairs should be very vulnerable to phonetic discontinuity. Fourth, we tried to select a wide range of splicing points. In repairs such as (5), (6), (7), (9), the first word of the repair proper (i.e. after the editing term) is the replacement for the trouble item. They are called instant repairs. In other repairs, such as (1) and (2), the speaker retraces to an earlier word so as to 'lead in' the replacing item. These retracings can be substantial (in number of words). The amount of retracing determines the distance between the splicing point and the point of trouble/replacement. Finally, care was taken to select repairs from a wide range of different speakers.

Guided by these five requirements we selected a set of repairs from the transcripts, i.e. without listening to the tapes. Subsequently we checked which of these were of sufficient acoustic quality to splice them by means of the Max-Planck speech editing system SPED. The surviving twenty-one candidates are listed in Table I.

The repairs are ordered in terms of increasing delay, and for each repair the places of splicing are marked by ' $/$ '. In other words, the stretch of speech between the first and the second occurrence of '/' was deleted in the splicing procedure. Also, the table lists the duration of this deleted stretch of speech, which is a measure for the delay between trouble item and replacement; the range is from 0.55 to $6.34 \mathrm{~s}$. Furthermore, the repairs' status as error/appropriateness and as marked/unmarked are given in the table. All repairs are from different speakers, except for the pairs 1 and 12, 5 and 6, 9 and 15, and for the triple $3,7,19$.

We (the author and Ger Desserjer - see acknowledgements) listened to the resulting utterances, and found out that up till item 18 they were all perfectly natural phonetically (items 3, 6, 9, 12, 15, and 18 were demonstrated during the presentation of this paper at the Congress of Phonetic Sciences, so that a wider audience could convince itself). It is especially important to notice that no loss of naturalness occurs for cases where the spliced interval exceeded the $1.3 \mathrm{~s}$. asymptotic value of the 'brief auditory trace' (items 9 through 18). This finding makes both echoic memory and working memory less likely loci for the preservation of the original utterance's relevant features. By default the results support the suspension theory: the speaker can interrupt the flow of speech at any moment, but hold the control structure in abeyance over substantial stretches of time which can be filled with other speech activities.

What is the limit for this persistence? Cases 19 to 21 suggest that the critical interval could be about 3 or $4 \mathrm{~s}$. Though case 21 sounds natural when spliced, both cases 19 (demonstrated at the Congress) and 20 are clearly unnatural. 
Table I. Repairs used for splicing. The parts between $\%$ ' and $\%$ ' are deleted. $(d=$ delay in seconds, $\mathrm{E}=$ error repair, $\mathrm{A}=$ appropriateness repair, $\mathrm{M}=$ prosodically marked, $\mathrm{U}=$ prosodically unmarked)

1. $(d=0.55, \mathrm{~A}, \mathrm{U})$ DAAR LINKS VAN / een blank of / EEN WIT KRUISPUNT (left thereof / a blank or / a white crossing point)

2. $(d=0.68, \mathrm{E}, \mathrm{M})$ VANUIT HET GROENE GA JE NAAR / links / RECHTS EN DAAR LIGT EEN EH GEEL KRUISPUNT

(from the green you go to the / left / right and there is a er yellow crossing point)

3. $(d=0.69, \mathrm{~A}, \mathrm{U})$ DAAR KUN JE ALLEEN MAAR RECHTSAF, DAN / gaan we n/DAN KOMEN WE BIJ HOEK ORANJE

(you can only go right, then / we go t- / then we come to corner orange)

4. $(d=0.80, \mathrm{E}, \mathrm{U})$ EN RECHTS VAN BLAUW IS EEN WEG / naar een grijze / NAAR EEN ROZE PUNT

(right of blue is a way / to a gray / to a pink point)

5. $(d=0.83, \mathrm{~A}, \mathrm{U})$ NAAR RECHTS / gaan we / LOPEN WE DOOD OP EEN ZWART KNOOPPUNT

(to the right / we go / we get stuck at a black node)

6. ( $d=0.90$, E, M) ANDERE MOGELIJKHEID VANUIT HET / groen-, eh / BRUINE PUNT LINKSAF NAAR EEN T-KRUISING GEEL

(other possibility from the / green, er / brown point left to a yellow T-crossing)

7. ( $d=1.05$, E, U) DAN GAAN WE EERST MAAR EVEN RECHTSAF NAAR PUNT / rood, eh sorry / ORANJE. DAT IS EEN EINDPUNT

(then we go first for a while to the right to point / red, er sorry / orange. That is an end point)

8. $(d=1.25, \mathrm{~A}, \mathrm{U})$ DE WEG OMHOOG / dat is een / DIE KOMT UIT BIJ EEN ZWARTE KRUISING

(the way up / that is a / that ends at a black crossing)

9. $(d=1.51, \mathrm{E}, \mathrm{M})$ DAAROP VOLGT / een horizon- nee / EEN VERTIKALE LIJN WAARBOVEN EEN WIT BOLLETJE ZIT

(therafter follows / a horizon- no / a vertical line above which is a white ball)

10. $(d=1.52, \mathrm{~A}, \mathrm{M})$ VANAF HET GELE KNOOPPUNT / gaan we $\mathrm{n}-/$ TREKKEN WE EEN VERBINDINGSSTREEPJE NAAR BENEDEN NAAR HET BLAUWE KNOOPPUNT

(from the yellow node / we go t- / we draw a connecting line downward to the blue node)

There is a lack of long-interval data in our corpus, and new data will be necessary to substantiate these values. Also, the persistence theory is clearly in need of further theoretical specification. One would like to know more about the precise nature of the stored code, about its sensitivity to interference etc.

Considering, finally, the close correspondence between the structure of repairs and the structure of coordination, it is of great interest to apply the same splicing test to coordinations, such as in (10):

\section{JOHN COOKED / and Mary ate / THE DINNER}

Will one find comparable values for the persistence of the control structure in these cases? 